Research Article

Open Access

Special Issue Dedicated to Charles R. Johnson

Mohammad Adm, Shaun Fallat*, Karen Meagher, Shahla Nasserasr, Sarah Plosker, and Boting Yang

\title{
Achievable multiplicity partitions in the inverse eigenvalue problem of a graph
}

https://doi.org/10.1515/spma-2019-0022

Received August 1, 2019; accepted December 2, 2019

Abstract: Associated to a graph $G$ is a set $\mathcal{S}(G)$ of all real-valued symmetric matrices whose off-diagonal entries are nonzero precisely when the corresponding vertices of the graph are adjacent, and the diagonal entries are free to be chosen. If $G$ has $n$ vertices, then the multiplicities of the eigenvalues of any matrix in $\mathcal{S}(G)$ partition $n$; this is called a multiplicity partition.

We study graphs for which a multiplicity partition with only two integers is possible. The graphs $G$ for which there is a matrix in $\mathcal{S}(G)$ with partitions $[n-2,2]$ have been characterized. We find families of graphs $G$ for which there is a matrix in $\mathcal{S}(G)$ with multiplicity partition $[n-k, k]$ for $k \geq 2$. We focus on generalizations of the complete multipartite graphs. We provide some methods to construct families of graphs with given multiplicity partitions starting from smaller such graphs. We also give constructions for graphs with matrix in $\mathcal{S}(G)$ with multiplicity partition $[n-k, k]$ to show the complexities of characterizing these graphs.

Keywords: inverse eigenvalue problem, multiplicity partition, adjacency matrix, minimum rank, distinct eigenvalues, graphs

MSC: 05C50, 15A18

\section{Introduction}

Let $G$ be a simple graph on $n$ vertices and consider the set $\mathcal{S}(G)$ of all possible real-valued weighted symmetric adjacency matrices associated to $G$, where the diagonal entries are free in that they may or may not be zero (the restriction herein to simple graphs avoids some unnecessary confusion when stating and proving results). The notation $\lambda_{i}^{\left(n_{i}\right)}$ is used to denote the eigenvalue $\lambda_{i}$ with multiplicity $n_{i}$. In this work, we order the eigenvalues according to their corresponding multiplicities. That is, a matrix $A \in \mathcal{S}(G)$ has spectrum $\sigma(A)=\left\{\lambda_{1}^{\left(n_{1}\right)}, \ldots, \lambda_{\ell}^{\left(n_{\ell}\right)}\right\}$, where $n_{1} \geq \cdots \geq n_{\ell}$ and the eigenvalues $\lambda_{i}$ are distinct. The list of eigenvalues could also be ordered according to their values, this is distinctly different from the ordering considered in this work; see $[10]$.

If $q(A)$ is the number of distinct eigenvalues of a symmetric matrix $A$, then for a given graph $G$, we let $q(G)=\min \{q(A): A \in \mathcal{S}(G)\}$, and refer to this parameter as the minimum number of distinct eigenvalues

\footnotetext{
Mohammad Adm: Department of Applied Mathematics and Physics, Palestine Polytechnic University, Hebron, Palestine Department of Mathematics and Statistics, University of Konstanz, Konstanz, Germany

*Corresponding Author: Shaun Fallat: Department of Mathematics and Statistics, University of Regina, Regina, SK, S4S 0A2, Canada

Karen Meagher: Department of Mathematics and Statistics, University of Regina, Regina, SK, S4S 0A2, Canada

Shahla Nasserasr, Sarah Plosker: Department of Mathematics and Statistics, University of Regina, Regina, SK, S4S 0A2,

Canada

Department of Mathematics and Computer Science, Brandon University, Brandon, MB R7A 6A9, Canada

Boting Yang: Department of Computer Science, University of Regina, Regina, SK, S4S 0A2, Canada
} 
of $G$. It is well-known that $q(G)=1$ if and only if $G$ has no edges, and $q(G)=n$ if and only if $G$ is a path on $n$ vertices; see [2]. Graphs with $q(G)=n-1$ are characterized in [4]. Some conditions for graphs attaining $q(G)=c$, for $c \in\{2, \ldots, n-2\}$ are given in [2]. For example, if a connected graph $G$ on at least three vertices has $q(G)=2$, then $G$ cannot have a cut edge (an edge whose deletion results in a disconnected graph), and any two non-adjacent vertices of $G$ must have at least two common neighbours (Lemma 4.2 and Corollary 4.5 of [2], respectively). Moreover, Corollary 3.6 of the same paper gives a construction for a graph on $n$ vertices satisfying $q(G)=c$ for any $1 \leq c \leq n$.

For positive integers $n_{1} \geq \cdots \geq n_{\ell}$, a partition $\left[n_{1}, n_{2}, \ldots, n_{\ell}\right]$ of $n$ is said to be achievable by $G$ if there exists an $A \in \mathcal{S}(G)$ such that the spectrum of $A$ is $\left\{\lambda_{1}^{\left(n_{1}\right)}, \ldots, \lambda_{\ell}^{\left(n_{\ell}\right)}\right\}$ for some set of distinct values $\lambda_{i}$. If $\left[n_{1}, n_{2}, \ldots, n_{\ell}\right]$ is a partition of $n$, we use the notation $M P\left(\left[n_{1}, n_{2}, \ldots, n_{\ell}\right]\right)$ to denote the set of all graphs on $n$ vertices for which the partition $\left[n_{1}, \ldots, n_{\ell}\right]$ of $n$ is achievable.

Given a graph, some natural questions arise. For example, what multiplicity partitions are achievable by a given graph or a family of graphs? This question is considered in $[1,3]$ where all achievable partitions are listed for all graphs on fewer than 6 vertices. Another question is if a certain multiplicity partition is achievable for a graph, is it possible to characterize any other multiplicity partitions that are also achievable for the graph? In particular, for two partitions $\left[n_{1}, n_{2}, \ldots, n_{\ell}\right]$ and $\left[\tilde{n}_{1}, \tilde{n}_{2}, \ldots, \tilde{n}_{m}\right]$ of $n$, when is $M P\left(\left[n_{1}, n_{2}, \ldots, n_{\ell}\right]\right) \subseteq$ $M P\left(\left[\tilde{n}_{1}, \tilde{n}_{2}, \ldots, \tilde{n}_{m}\right]\right)$ ?

Another natural approach is to characterize the graphs in $M P\left(\left[n_{1}, \ldots, n_{\ell}\right]\right)$ for some partition $\left[n_{1}, \ldots, n_{\ell}\right]$. This has been answered for a limited number of partitions. For example, the main result in [14] is that every connected graph on $n$ vertices is in $M P([1,1, \ldots, 1])$. The only graph that is in $M P([n])$ is the graph on $n$ vertices with no edges. The set $M P([n-1,1])$ is exactly the set of graphs on $n$ vertices that have one connected component that is a complete graph and the remaining components are isolated vertices (this includes the graph on $n$ vertices with no edges). The graphs in $M P([n-2,2])$ have also been exactly characterized; this characterization is given in Lemma 2.3 and see any of $[7,12,13,15])$ for a proof.

The questions and studies mentioned above all fit under the general umbrella of the inverse eigenvalue problem (IEVP) of a graph, specifically looking at the achievable multiplicities of the eigenvalues in the IEVP. Many authors have provided partial answers to these questions; see $[2-4,8,10,13]$. Here, we add to the growing body of work, focusing on joins, complete multipartite graphs and the sets $M P([n-k, k])$ for some $k$ with $k \leq\left\lfloor\frac{n}{2}\right\rfloor$.

\section{Graphs with two Distinct Eigenvalues}

Our goal is to consider the graphs $G$ with $q(G)=2$; any such graph on $n$ vertices achieves a bipartition [ $n-k, k]$ for some $k=1,2, \ldots,\left\lfloor\frac{n}{2}\right\rfloor$. The initial results that we state show that this is a very large set of graphs.

The join of two graphs $G$ and $H$ is the graph $G \vee H$ on vertex set $V(G) \cup V(H)$, where all edges of $G$ and $H$ are preserved, and edges are added to make every vertex of $G$ incident to every vertex of $H$. The following theorem by Monfared and Shader [14, Theorem 5.2] gives a sufficient condition for the minimum number of distinct eigenvalues to be 2 .

Theorem 2.1. Let $G$ and $H$ be two connected graphs on $n$ vertices. Then $q(G \vee H)=2$.

In [11] a large number of graphs are shown to admit only two distinct eigenvalues. Theorem 3.2 of [11] proves for a tree $T$ that $q(\bar{T})=2$, unless $T$ is $P_{4}$, or in one of two families of trees. Further, Theorem 2.5 of the same paper proves that many bipartite graphs $G$ have the property $q(\bar{G})=2$. This gives another large and diverse family of graphs with only 2 distinct eigenvalues.

The results in [14] and [11] indicate that the collection of graphs with only two distinct eigenvalues is very large and likely very difficult to characterize. Thus rather than trying to characterize all graphs $G$ with $q(G)=$ 2, we define the minimal multiplicity bipartition $M B(G)$ to be the least integer $k \leq\left\lfloor\frac{n}{2}\right\rfloor$ such that $G$ achieves 
the multiplicity bipartition $[n-k, k]$ (hence if $k=M B(G)$, then $G \in M P([n-k, k])$ but $G \notin M P([n-m, m])$ for any $m<k$ ). If $M B(G)=k$, then we say $G$ has multiplicity bipartition $k$. The multiplicity bipartition is only defined for graphs that admit only two distinct eigenvalues and if $G$ has $n$ vertices, then $M B(G) \leq n / 2$.

It is easy to note that if $\mathcal{S}(G)$ has a matrix with only two distinct eigenvalues, then for any two distinct real numbers there exists a matrix in $\delta(G)$ such that its spectrum consists of these two real numbers.

For an $m \times n$ matrix $A$, the notation $A[\alpha \mid \beta]$ is used to denote the submatrix of $A$ lying in rows indexed by $\alpha$ and columns indexed by $\beta$. We let $J_{n \times m}\left(J_{n}\right)$ denote the $n \times m(n \times n)$ matrix with all entries equal to one, and we let $O_{n}$ denote the $n \times n$ zero matrix.

Lemma 2.2. Let $G$ be a graph on $n$ vertices with $q(G)=2$. Then $G \in M P([n-k, k])$ if and only if there is an $A \in \mathcal{S}(G)$ with

$$
A=u_{1} u_{1}^{T}+u_{2} u_{2}^{T}+\cdots+u_{k} u_{k}^{T},
$$

where $k \leq\left\lfloor\frac{n}{2}\right\rfloor$ and $\left\{u_{1}, u_{2}, \ldots, u_{k}\right\}$ is a set of orthonormal vectors in $\mathbb{R}^{n}$.

Proof. Assume that $q(G)=2$ and $G \in M P([n-k, k])$, then there is a matrix $A \in \mathcal{S}(G)$ with $\sigma(A)=\left\{0^{(n-k)}, 1^{(k)}\right\}$. Therefore, $A$ can be written as

$$
A=V\left(I_{k} \oplus O_{n-k}\right) V^{T}=U U^{T},
$$

where $V$ is a unitary matrix, $U=V[1, \ldots, n \mid 1, \ldots, k]$ and $O_{n-k}$ is the $(n-k) \times(n-k)$ all zeros matrix. Let $U=\left(u_{1}, u_{2}, \ldots, u_{k}\right)$ where $u_{i} \in \mathbb{R}^{n}, i=1, \ldots, k$. Each $u_{i}$ is a column of $V$, so they are orthonormal. Moreover, $A=u_{1} u_{1}^{T}+u_{2} u_{2}^{T}+\cdots+u_{k} u_{k}^{T}$.

Conversely, assume there is a matrix $A=u_{1} u_{1}^{T}+u_{2} u_{2}^{T}+\cdots+u_{k} u_{k}^{T}$, where $\left\{u_{1}, u_{2}, \ldots, u_{k}\right\}$ is a set of orthonormal vectors in $\mathbb{R}^{n}$. Then the spectrum for $A$ is $\left\{0^{(n-k)}, 1^{(k)}\right\}$, so $G \in M P([n-k, k])$.

We summarize the known characterizations of graphs with given minimal multiplicity bipartitions in the following lemma.

Lemma 2.3. Let $G$ be a connected graph on $n$ vertices. Then

1. $M B(G)=1$ if and only if $G$ is the complete graph, $K_{n}$.

2. $M B(G)=2$ if and only if

$$
G=\left(K_{p_{1}} \cup K_{q_{1}}\right) \vee\left(K_{p_{2}} \cup K_{q_{2}}\right) \vee \cdots \vee\left(K_{p_{k}} \cup K_{q_{k}}\right),
$$

for non-negative integers $p_{1}, \ldots, p_{k}, q_{1}, \ldots, q_{k}$ with $k>1$, and $G$ is not isomorphic to either one of a complete graph or $\left(K_{p_{1}} \cup K_{q_{1}}\right) \vee K_{1}$.

3. If $M B(G)=k$, then $G$ does not have an independent set (a set of vertices for which no two are adjacent) of size $k+1$ or more.

Proof. The first statement is trivial. The second statement has appeared in [7, 12, 13, 15].

The third statement is known (for example, there is a proof in [13]), but we include a proof for completeness. From Lemma 2.2 there is a matrix $A \in \mathcal{S}(G)$ with

$$
A=u_{1} u_{1}^{T}+u_{2} u_{2}^{T}+\cdots+u_{k} u_{k}^{T}
$$

where $u_{i}, i=1,2, \ldots, k$, are orthonormal vectors. Let $U=\left[u_{1}, u_{2}, \ldots, u_{k}\right]$ where $u_{i} \in \mathbb{R}^{n}, i=1, \ldots, k$. If $G$ has an independent set of size $k+1$, then the rows of $U$ form $k+1$ orthogonal vectors in $\mathbb{R}^{k}$, which is impossible. Hence there is no independent set of size $k+1$.

The following lemma indicates that for a connected graph $G$ with $q(G)=2$, if the union of the pairwise common neighbourhood of an independent set of vertices is not empty, then it cannot be too small. 
Lemma 2.4 (Theorem 4.4, [2]). Consider a connected graph $G$ with $q(G)=2$, and let $S$ be an independent set of vertices. If $\cup_{v_{i}, v_{j} \in S}\left(N\left(v_{i}\right) \cap N\left(v_{j}\right)\right)$ is not empty, then

$$
|S| \leq\left|\cup_{i, j \in S}\left(N\left(v_{i}\right) \cap N\left(v_{j}\right)\right)\right| .
$$

For a given graph $G$, the minimum rank among all matrices (positive semidefinite matrices) in $\mathcal{S}(G)$ is denoted by $\operatorname{mr}(G)\left(\mathrm{mr}_{+}(G)\right)$. If a graph $G$ on $n$ vertices has $q(G)=2$ and $M B(G)=k$, from Lemma 2.2 then there is a matrix $A \in \mathcal{S}(G)$ with spectrum $\left\{0^{(n-k)}, 1^{(k)}\right\}$. This implies the following lemma.

Lemma 2.5. If $G$ is any graph with $q(G)=2$, then $\operatorname{mr}(G) \leq \mathrm{mr}_{+}(G) \leq M B(G)$.

The parameter $\operatorname{mr}(G)$ has been extensively studied [9]; and any lower bound on the minimum rank or the minimum positive semidefinite rank of a graph, is also a lower bound on the minimal multiplicity bipartition of the graph. For example, we may use the above bound on minimum rank together with $[9$, Obs. 1.6] to deduce the next result.

Lemma 2.6. If $G$ is any graph with $M B(G)=k$, then any induced path of $G$ has length no more than $k$.

\section{Complete Multipartite Graphs}

As is standard, we use the notation $K_{p_{1}, p_{2}, \ldots, p_{\ell}}$ for the complete $\ell$-partite graph, where $\ell$ is a positive integer. The set of vertices is partitioned into $\ell$ disjoint parts $V_{1} \cup V_{2} \cup \cdots \cup V_{\ell}$; part $V_{i}$ has $p_{i}$ vertices for $i \in\{1, \ldots, \ell\}$; no two vertices from a part are adjacent, while any two vertices from different parts are adjacent.

In this section we show that the value of $q\left(K_{p_{1}, p_{2}, \ldots, p_{\ell}}\right)$ is either 2 or 3, depending on the size of its parts.

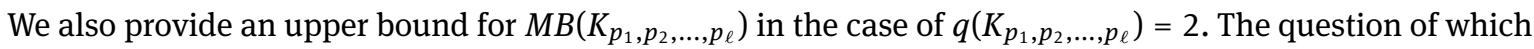
other multiplicity partitions can be achieved by a complete multipartite graph remains open.

In an unpublished manuscript (see [5]), it is shown that any complete multipartite graph $K_{p_{1}, p_{2}, \ldots, p_{\ell}}$ satisfies $q\left(K_{p_{1}, p_{2}, \ldots, p_{\ell}}\right) \leq 3$. The basic idea employed in the proof of this inequality is to note that the matrix $B=\left[b_{u, v}\right]$ with entries defined as

$$
b_{u, v}= \begin{cases}0 & \text { if } u, v \in V_{i}, \\ \frac{1}{\sqrt{p_{i} \bar{p}_{j}}} & \text { if } u \in V_{i}, v \in V_{j}, \text { and } i \neq j .\end{cases}
$$

satisfies $B \in \mathcal{S}\left(K_{p_{1}, p_{2}, \ldots, p_{\ell}}\right)$ and $q(B)=3$. Furthermore, it can be easily verified that the eigenvalues of $B$ are $\{-1,0, \ell-1\}$ with multiplicities $\ell-1, \sum_{i=1}^{\ell}\left(p_{i}-1\right), 1$, respectively. Thus if follows that $K_{p_{1}, p_{2}, \ldots, p_{\ell}} \in$ $M P\left(\left[\sum_{i=1}^{\ell}\left(p_{i}-1\right), \ell-1,1\right]\right)$.

There are several known results for the partitions that are achievable for the complete multipartite graphs; we list them in the following lemma.

Lemma 3.1. For positive integers $\ell, p_{i}, q_{i}$ with $i=1,2, \ldots, \ell$

1. $q\left(K_{p_{1}, p_{2}, \ldots, p_{\ell}}\right) \in\{2,3\}$.

2. $q\left(K_{p_{1}, q_{1}}\right)= \begin{cases}2 & \text { if } p_{1}=q_{1} \\ 3 & \text { otherwise. }\end{cases}$

3. If $p_{1}+p_{2}+\cdots+p_{\ell}=q_{1}+q_{2}+\cdots+q_{\ell^{\prime}}$ for $\ell, \ell^{\prime} \geq 2$, then

$$
q\left(K_{p_{1}, p_{2}, \ldots, p_{\ell}, q_{1}, q_{2}, \ldots, q_{\ell^{\prime}}}\right)=2 .
$$

4. If $p_{2}+\cdots+p_{\ell}<p_{1}$, then $q\left(K_{p_{1}, p_{2}, \ldots, p_{\ell}}\right)=3$.

Proof. The first statement is from [2]. The second statement follows from Theorem 2.1. 
To observe that the third statement holds, note that $p_{1}+p_{2}+\cdots+p_{\ell}=q_{1}+q_{2}+\cdots+q_{\ell^{\prime}}$ for $\ell$, $\ell^{\prime} \geq 2$ implies that $K_{p_{1}, p_{2}, \ldots, p_{\ell}, q_{1}, q_{2}, \ldots, q_{\ell^{\prime}}}$ is isomorphic to the join of $K_{p_{1}, p_{2}, \ldots, p_{\ell}}$ and $K_{q_{1}, q_{2}, \ldots, q_{\ell^{\prime}}}$, and hence satisfies $q\left(K_{p_{1}, p_{2}, \ldots, p_{\ell}, q_{1}, q_{2}, \ldots, q_{\ell^{\prime}}}\right)=2$ by Theorem 2.1 .

To see that the last statement holds, assume that $p_{2}+\cdots+p_{\ell}<p_{1}$, and set $n=p_{1}+p_{2}+\cdots+p_{\ell}$; this implies $n-p_{1}<p_{1}$. If $q\left(K_{p_{1}, p_{2}, \ldots, p_{\ell}}\right)=2$, then $K_{p_{1}, p_{2}, \ldots, p_{\ell}} \in M P([n-k, k])$ with $p_{1} \leq k \leq n-k$ (by Statement (3) of Lemma 2.3). Hence $p_{1} \leq k \leq n-k \leq n-p_{1}$, implying $2 p_{1} \leq n$, which is a contradiction. Hence $q\left(K_{p_{1}, p_{2}, \ldots, p_{\ell}}\right) \geq 3$. Finally, the equality follows from the work in the unpublished manuscript [5].

Continuing with the complete multipartite graph, we consider the particular case $p_{1} \leq p_{2}+\cdots+p_{\ell}$. Along

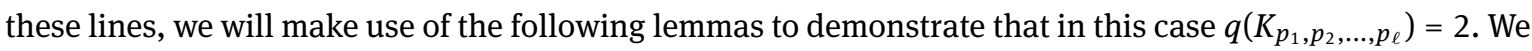
begin by stating a technical result that is a special case of [6, Lemma 10] (in the notation of [6], we are setting $q=0$ and $p=k \geq 2$ ).

Lemma 3.2. Let $k \geq 2$, and $M_{1}$ and $M_{2}$ be matrices that have $k$ rows and no zero columns. Then there exists a $k \times k$ matrix $R$ such that $R^{T} R=I_{k}$ and $M_{1}^{T} R M_{2}$ has no zero entries.

Lemma 3.3. For any graph $G$ with no isolated vertices and for any number $d$ such that $\operatorname{mr}_{+}(G) \leq d \leq|V(G)|$, there exist vectors $q_{1}, \ldots, q_{d}$ such that $\sum_{i} q_{i} q_{i}^{T} \in \mathcal{S}(G)$ and each $q_{i}, i=1, \ldots, d$, is entry-wise nonzero.

Proof. Since for the given parameter $d$ we have $\operatorname{mr}_{+}(G) \leq d \leq|V|$, it follows that there is an $A \in \mathcal{S}_{+}(G)-$ the set of positive semidefinite matrices in $\mathcal{S}(G)$-with $\operatorname{rank}(A)=d$. Since $G$ has no isolated vertices, $A$ has no zero rows or columns. Since $A$ is positive semidefinite, $A$ can be written as $A=U U^{T}$, for some $n \times d$ matrix $U$. If $U=\left[u_{1}, u_{2}, \ldots, u_{d}\right]$, where $u_{1}, u_{2}, \ldots, u_{d}$ are the columns of $U$, then we may assume that $u_{1}, u_{2}, \ldots, u_{d}$ are mutually orthogonal vectors in $\mathbb{R}^{n}$.

Set $M_{1}=U^{T}$, and $M_{2}=I_{d}$; each of these matrices have $d$ rows and do not have any zero columns (this follows since $A$ has no zero rows). Hence, by Lemma 3.2, there exists an orthogonal $d \times d$ matrix $R$ such that $M_{1}^{T} R M_{2}=U R$ has no zero entries. Let $Q=U R=\left[q_{1}, q_{2}, \ldots, q_{d}\right]$ then

$$
Q Q^{T}=U R R^{T} U^{T}=U U^{T}=A \in \mathcal{S}(G) .
$$

as needed.

The next result, in which a slightly more general version originally appeared in [5], is a technical result concerning a bound on the minimum semidefinite rank of joins of graphs. This result is needed to complete our study on the minimum number of distinct eigenvalues of the complete multipartite graph and establish a bound on the corresponding minimal multiplicity bipartition. We provide a proof here for completeness of exposition. In this proof $\mathbf{1}_{s}$ is used to denote the vector in $\mathbb{R}^{s}$ with all entries equal to one. Similarly, $\mathbf{0}_{s}$ is used to denote the vector in $\mathbb{R}^{s}$ with all entries equal to zero and $O$ is the all zeros matrix, the size will be clear from context.

Lemma 3.4. Consider the graph $G$ with no isolated vertices and positive integers $d, s_{1}, s_{2}, \ldots, s_{d}$. If $0<$ $\operatorname{mr}_{+}(G) \leq d \leq|V(G)|$, then $q\left(G \vee\left(K_{s_{1}} \cup K_{s_{2}} \cup \cdots \cup K_{s_{d}}\right)\right)=2$; moreover, $M B\left(G \vee\left(K_{s_{1}} \cup K_{s_{2}} \cup \cdots \cup K_{s_{d}}\right)\right)=d$.

Proof. Let $H=G \vee\left(K_{s_{1}} \cup K_{s_{2}} \cup \cdots \cup K_{s_{d}}\right)$, and $s=\sum_{i=1}^{d} s_{i}$. We construct a matrix $B \in \mathcal{S}(H)$ with $\sigma(B)=\{0, \beta\}$, where the eigenvalue 0 has multiplicity $|V(H)|-d$ and the eigenvalue $\beta$ has multiplicity $d$. Using Lemma 3.3, there are entry-wise nonzero vectors $q_{1}, \ldots, q_{d}$ such that $A=\sum_{i=1}^{d} q_{i} q_{i}^{T} \in \mathcal{S}(G)$. Construct vectors $v_{1}, \ldots, v_{d}$ as follows

$$
v_{1}=\left[\frac{q_{1}}{\frac{\alpha_{1} \mathbf{1}_{s_{1}}}{\mathbf{0}_{s-s_{1}}}}\right], v_{2}=\left[\frac{q_{2}}{\frac{\mathbf{0}_{\mathbf{s}_{1}}}{\alpha_{2} \mathbf{1}_{s_{2}}}}\right], \ldots, v_{d}=\left[\begin{array}{c}
q_{d} \\
\frac{\mathbf{0}_{s-s_{d}}}{\alpha_{s-s_{1}-s_{2}}}
\end{array}\right]
$$

the value of $\alpha_{i}$ will be determined. Let $q_{i}^{T}=\left[\begin{array}{lll}q_{1, i}, & q_{2, i}, & \ldots,\end{array} q_{n, i}\right]$ for $i=1, \ldots, d$ where $|V(G)|=n$. 
For $j=2, \ldots, d+1$, let $B_{1, j}=\left[q_{j-1}, q_{j-1}, \ldots, q_{j-1}\right]$ be the $n \times s_{j-1}$ matrix with all columns equal to $q_{j-1}$. The matrix $B=\sum_{i=1}^{d} v_{i} v_{i}^{T}$ has the following form:

$$
B=\left[\begin{array}{c|cccc}
A & \alpha_{1} B_{1,2} & & \ldots & \alpha_{d} B_{1, d+1} \\
\hline \alpha_{1} B_{1,2}^{T} & \alpha_{1}^{2} J_{s_{1} \times S_{1}} & 0 & \ldots & O \\
& 0 & \alpha_{2}^{2} J_{S_{2} \times s_{2}} & & 0 \\
\vdots & & & & \vdots \\
& \vdots & & \ddots & 0 \\
\alpha_{d} B_{1, d+1}^{T} & 0 & \ldots & O & \alpha_{d}^{2} J_{s_{d} \times s_{d}}
\end{array}\right]
$$

Now, for a positive number $\beta>\max \left\{\left\|q_{i}\right\|^{2}, i=1, \ldots, d\right\}$, set $\alpha_{i}=\sqrt{\frac{\beta-\left\|q_{i}\right\|^{2}}{s_{i}}}$. Then $B \in \mathcal{S}(H) \operatorname{and} \operatorname{rank}(B)=$ $d$, which implies the eigenvalue zero has multiplicity $|V(H)|-d$. On the other hand, $B v_{i}=\beta v_{i}$ for each $i=1,2, \ldots, d$, and the vectors $v_{1}, \ldots, v_{d}$ are linearly independent. Thus $\sigma(B)=\{0, \beta\}$ with the desired multiplicities, and since $H$ has at least $d$ independent vertices, $d \leq M B(H)$.

Note that, in Lemma 3.4, if $G$ has $\ell$ isolated vertices, then these vertices form an independent set. By Lemma 2.4, in order for $q(G)=2$, the union of the mutual common neighbours of an independent set cannot have more than $\ell$ elements; therefore, $\ell \leq \sum_{i}^{d} s_{i}$. Moreover, if $d=s_{1}=1$ and $G$ has isolated vertices, then there is a unique path from a vertex of $G$ to an isolated vertex of $G$ using the vertex of $K_{S_{1}}$, which implies the graph cannot have only two distinct eigenvalues. It is unclear if the statement of Lemma 3.4 holds in other cases when $G$ has $\ell$ isolated vertices.

The fact that the minimum number of distinct eigenvalues of complete multipartite graphs is at most three is a special case of Lemma 3.4. The next result shows Statement 4 of Lemma 3.1 gives the only family of complete multipartite graphs that do not have only two distinct eigenvalues.

Corollary 3.5. Any complete multipartite graph $H=K_{p_{1}, p_{2}, \ldots, p_{\ell}}$ with $p_{1} \geq p_{2} \geq \cdots \geq p_{\ell}, p_{1} \leq p_{2}+\cdots+p_{\ell}$, and $\ell \geq 3$ achieves two distinct eigenvalues, and $M B(H)=p_{1}$.

Proof. Note that

$$
H=\underbrace{\left(K_{1} \cup K_{1} \cup \cdots \cup K_{1}\right)}_{p_{1} \text { times }} \vee K_{p_{2}, \ldots, p_{\ell}} .
$$

Let $G=K_{p_{2}, \ldots, p_{\ell}}, d=p_{1}$ and $s_{1}=s_{2}=\cdots=s_{d}=1$. Then $\mathrm{mr}_{+}(G)=p_{2} \leq p_{1}=d$ (see [17, Prop. 3.4]). Using Lemma 3.4, $q(H)=2$ with $M B(H)=p_{1}$.

A specific case of Corollary 3.5 is when all the parts have the same sizes.

Corollary 3.6. Let $G=K_{k, k, \ldots, k}, k \geq 2$ be the complete multipartite graph on $n$ vertices. Then $q(G)=2$ and $M B(G)=k$.

For a vertex $v$ in a graph $G$, a new graph $G^{\prime}$ can be constructed by cloning (or duplicating) $v$. The graph $G^{\prime}$ has vertex set $V\left(G^{\prime}\right)=V(G) \cup\left\{v^{\prime}\right\}$ and edge set $E\left(G^{\prime}\right)=E(G) \cup\left\{v^{\prime} u ; u \in N[v]\right\}$, where $N[v]$ is the closed neighbourhood of $v$ (that is, a neighbourhood of $v$ containing $v$ ). It turns out that cloning a vertex of a graph $G$ with $M B(G)=k$ results in a graph $G^{\prime}$ with $M B\left(G^{\prime}\right) \leq k$. The following proposition is proved in Theorem 6.3 of [12], it is also implied by Corollary 4 of [1]. In [12], this is used to characterize graphs $G$ with $M B(G)=2$ by constructing minimal such graphs (these are $K_{1}, K_{1} \cup K_{1}, K_{2,1}, K_{2,2, \ldots, 2}$ and $K_{2,2, \ldots, 2,1}$ ) and constructing all the other such graphs by cloning vertices in the minimal graphs.

Proposition 3.7. Let $G$ be a graph with $G \in M P([n-k, k])$. If $H$ is obtained from $G$ by cloning a vertex in $G$, then $H \in M P([n-k+1, k])$. 
Suppose $G$ is a graph with $q(G)=2$. If $H$ is obtained from $G$ by cloning a vertex, then $M B(H) \leq M B(G)$. It is not clear if this inequality is ever strict. By cloning vertices in $K_{k, k, \ldots, k}$, where $k \geq 2$, we have the following consequence, reminiscent of Lemma 2.3.

Corollary 3.8. If $G=\bigvee_{i=1}^{l}\left(\cup_{j=1}^{k} K_{a_{i, j}}\right)$ where $k \geq 2, i=1,2, \ldots, l, j=1,2, \ldots, k$, and $a_{i, j}$ are positive integers, then $q(G)=2$ and $M B(G)=k$.

Unlike the case where $k=2$, for general $k$ the previous corollary does not characterize all the graphs in $M P([n-k, k])$. In Section 4, several graphs in $M P([n-k, k])$ that are not included in Corollary 3.8 are given.

Theorem 3.9. Let $G$ and $H$ be two graphs with no isolated vertices. Further assume that $q(G)=q(H)=2$ with $M B(G)=M B(H)$. Then $q(G \vee H)=2$ and $M B(G \vee H) \leq M B(G)(=M B(H))$.

Proof. Assume $M B(G)=M B(H)=k$. If $k=1$, there is nothing to prove, so assume $k \geq 2$. Let $n_{1}$ be the number of vertices in $G$ and $n_{2}$ the number of vertices in $H$. Let $A \in \mathcal{S}(G)$ be such that $\sigma(A)=\left\{0^{\left(n_{1}-k\right)}, 1^{(k)}\right\}$ and let $B \in \mathcal{S}(H)$ be such that $\sigma(B)=\left\{0^{\left(n_{2}-k\right)}, 1^{(k)}\right\}$. By Schur's Theorem, there exists orthogonal matrices $Q_{1}$ and $Q_{2}$ such that

$$
A=Q_{1}^{T}\left(I_{k} \oplus O_{n_{1}-k}\right) Q_{1} \quad \text { and } \quad B=Q_{2}^{T}\left(I_{k} \oplus O_{n_{2}-k}\right) Q_{2} .
$$

Let

$$
M_{1}=Q_{1}\left[1, \ldots, k \mid 1, \ldots, n_{1}\right] \text { and } M_{2}=Q_{2}\left[1, \ldots, k \mid 1, \ldots, n_{2}\right],
$$

so $A=M_{1}^{T} M_{1}$ and $B=M_{2}^{T} M_{2}$. This also implies that $M_{1}$ has no zero columns, since otherwise $A$ would have a row and column of zeros which would imply that $G$ would have an isolated vertex. Similarly, $M_{2}$ has no zero columns.

By Lemma 3.2, there exists a $k \times k$ matrix $R$ such that $R^{T} R=I_{k}$ and $M_{1}^{T} R M_{2}$ has no zero entries. Define $C$ as follows:

$$
\begin{aligned}
C=\left[\begin{array}{c}
M_{1}^{T} \\
M_{2}^{T} R^{T}
\end{array}\right]\left[\begin{array}{ll}
M_{1} & R M_{2}
\end{array}\right] & =\left[\begin{array}{cc}
M_{1}^{T} M_{1} & M_{1}^{T} R M_{2} \\
M_{2}^{T} R^{T} M_{1} & M_{2}^{T} R^{T} R M_{2}
\end{array}\right] \\
& =\left[\begin{array}{cc}
A & M_{1}^{T} R M_{2} \\
M_{2}^{T} R^{T} M_{1} & B
\end{array}\right] .
\end{aligned}
$$

Hence $C$ is positive semidefinite and $C \in \mathcal{S}(G \vee H)$ since $M_{1}^{T} R M_{2}$ is an entry-wise nonzero matrix. It is easy to note that $C$ has rank $k$ since $\left[M_{1} R M_{2}\right]$ has a full-row rank. Therefore, null $(C)=n_{1}+n_{2}-k$. Moreover, $C^{2}=2 C$ which implies $\sigma(C)=\left\{0^{\left(n_{1}+n_{2}-k\right)}, 2^{(k)}\right\}$. Hence $q(G \vee H)=2$ since $C \in \mathcal{S}(G \vee H)$ and $q(G \vee H)>1$.

In Theorem 3.9, where $k=M B(G)=M B(H)$, if $k=1$, then the inequality $M B(G \vee H) \leq 1$ cannot be strict, because $M B(G \vee H) \geq 1$ for all graphs where it is defined. Similarly, if $k=2$, then the inequality $M B(G \vee H) \leq 2$ cannot be strict, because $M B(G \vee H)=1$ implies that $G \vee H$ is a complete graph with possibly isolated vertices, which is a contradiction. Moreover, if $k=3$, then the inequality $M B(G \vee H) \leq 3$ cannot be strict, because $M B(G \vee H)=1,2$ implies that $G \vee H$ is either a complete graph with possibly isolated vertices, or a graph characterized in Lemma 2.3 (2), either case is a contradiction.

For cases of $k \geq 4$ in Theorem 3.9, it is still unclear if a strict inequality can hold in $M B(G \vee H) \leq k$. We suspect, that in fact, equality among $M B(G \vee H)=M B(G)=M B(H)$ holds under the hypothesis of Theorem 3.9. A related matter is to determine if a version of Theorem 3.9 still holds in the case when $M B(G) \neq M B(H)$. It turns out that the requirement of $M B(G)=M B(H)$ is essential in concluding that $q(G \vee H)=2$ as in Theorem 3.9. Consider the following example. Let $G=Q_{6}$ (the 6-dimensional hypercube), and let $S=\{x, y, z\}$ be the independent set of vertices in $G$ consisting of $x=(000000), y=(010101)$, and $z=(111111)$. Also observe that there are no common neighbours among any pair of vertices from $S$ in $G$. Let $H=P_{2}$. Then we have $q(G)=q(H)=2$, and $M B(G) \geq 3$ and $M B(H)=1$. However, in the graph $G \vee H$, using the independent set $S$, it is easy to deduce that the condition of Lemma 2.4 fails. Hence $q(G \vee H)>2$.

In fact, this idea can be easily generalized as follows: Suppose $G$ is a graph with $q(G)=2$ that contains an independent set of vertices $S=\left\{v_{1}, v_{2}, \ldots, v_{k}\right\}$ in which $\cup_{v_{i}, v_{j} \in S}\left(N\left(v_{i}\right) \cap N\left(v_{j}\right)\right)=\emptyset$. Then for any graph $H$ 
with $q(H)=2$ and $|H|<k$, we have $q(G \vee H)>2$. To see this, it is enough to observe that in the graph $G \vee H$ we have

$$
\left|\cup_{v_{i}, v_{j} \in S}\left(N\left(v_{i}\right) \cap N\left(v_{j}\right)\right)\right|=|H|<|S|,
$$

and hence the condition of Lemma 2.4 fails to hold.

We also note that the assumption of no isolated vertices in Theorem 3.9 is possibly a stronger condition than is in fact necessary; this assumption is used to ensure that the matrix $M_{2}$ in the proof has no zero columns. For instance, in the next result, which is a weaker version of Lemma 3.4, all the vertices of the second graph are isolated vertices. The proof of Lemma 3.10 is the same as the proof of Theorem 3.9, except that the matrix $B$ is replaced with the identity matrix. We denote the graph on $k$ vertices with no edges by $\overline{K_{k}}$.

Lemma 3.10. Let $G$ be a graph with no isolated vertices and $q(G)=2$ with $G \in M P([n-k, k])$ for some $k \geq 2$. Then $q\left(G \vee \overline{K_{k}}\right)=2$ with $M B\left(G \vee \overline{K_{k}}\right) \leq k$.

Note that the multiplicity bipartition $[n-k, k]$ for regular complete multipartite graphs $K_{k, k, \ldots, k}$ can also be obtained from the proof of Theorem 3.9 and induction. We skip the details since a more general result is shown in Corollary 3.5.

It is also interesting to note that the minimum number of distinct eigenvalues of the join of two graphs can be large.

Lemma 3.11. For any graph $G, q\left(G \vee K_{1}\right) \geq\left\lceil\frac{q(G)+1}{2}\right\rceil$.

Proof. The eigenvalues for any matrix in $\mathcal{S}(G)$ interlace the eigenvalues any matrix $\mathcal{S}\left(G \vee K_{1}\right)$.

The next theorem is the main result of [14].

Theorem 3.12 (Theorem 4.3 [14]). Let $G$ be a connected graph on $n$ vertices and let $\lambda_{1}, \lambda_{2}, \ldots, \lambda_{n}$ be distinct real numbers. Then there exists a real symmetric matrix $A \in \mathcal{S}(G)$ with eigenvalues $\lambda_{1}, \lambda_{2}, \ldots, \lambda_{n}$ such that none of the eigenvectors of $A$ has a zero entry.

Lemma 3.13. Let $G$ be a connected graph on $n \geq 2$ vertices. Then $q\left(G \vee \overline{K_{n}}\right)=2$ and $M B\left(G \vee \overline{K_{n}}\right)=n$.

Proof. Since $G$ is a connected graph, by Theorem 3.12 there exists a matrix $A \in \mathcal{S}(G)$ with positive distinct eigenvalues

$$
\lambda_{1}>\lambda_{2}>\cdots>\lambda_{n}
$$

and corresponding entry-wise nonzero unit eigenvectors $v_{1}, \ldots, v_{n}$ such that

$$
A=V^{T} \Lambda V=U^{T} U
$$

where $U=\Lambda^{\frac{1}{2}} V, \Lambda=\operatorname{diag}\left(\lambda_{1}, \ldots, \lambda_{n}\right)$, and $V^{T}=\left[v_{1}, \ldots, v_{n}\right]$. The rows of $U$ are orthogonal since $V$ is unitary. Let $C$ be the $n \times 2 n$ matrix $C=\left[\begin{array}{ll}D & U\end{array}\right]$, where

$$
D=\left[\begin{array}{cccc}
a_{1} & 0 & \ldots & 0 \\
0 & a_{2} & \ldots & 0 \\
\vdots & & \ddots & \vdots \\
0 & 0 & \ldots & a_{n}
\end{array}\right],
$$

where the scalars $a_{i}(i=1,2, \ldots, n)$ are to be determined. Since $U$ is an entry-wise nonzero matrix, if each $a_{i}$ is also nonzero, then

$$
C^{T} C=\left[\begin{array}{cc}
D^{2} & D U \\
U^{T} D & U^{T} U
\end{array}\right] \in \mathcal{S}\left(G \vee \overline{K_{n}}\right) .
$$

Further, the rows of $C$ are orthogonal and so

$$
C C^{T}=\left[\begin{array}{cccc}
\alpha_{1} & 0 & \ldots & 0 \\
0 & \alpha_{2} & \ldots & 0 \\
\vdots & & \ddots & \vdots \\
0 & 0 & \ldots & \alpha_{n}
\end{array}\right],
$$


where $\alpha_{i}=a_{i}^{2}+\lambda_{i}^{2}, i=1, \ldots, n$. Therefore, the eigenvalues of $C C^{T}$ are $\alpha_{i}, i=1, \ldots, n$. The values $a_{i}$ can be set so that they are all strictly positive, and $\alpha_{i}$ for all $i=1, \ldots, n$ are equal to some $\lambda_{0}>\lambda_{1}^{2}$. Then the spectrum of $C^{T} C$ is 0 with multiplicity $n$, and $\lambda_{0}$ also with multiplicity $n$. This implies that $q\left(G \vee \overline{K_{n}}\right)=2$ and $M B\left(G \vee \overline{K_{n}}\right) \leq n$. Finally, the vertices in $\overline{K_{n}}$ form an independent set of size $n$, and so by Statement 3 of Lemma 2.3, it follows that $M B\left(G \vee \overline{K_{n}}\right)=n$.

The same proof can be used to prove the following result.

Lemma 3.14. Let $G$ be a connected graph on $n \geq 2$ vertices. Then $q\left(G \vee \bar{K}_{n-1}\right)=2$ and $M B\left(G \vee \bar{K}_{n-1}\right)=n-1$.

Proof. As in the proof of the previous lemma, there exists a matrix $A \in \mathcal{S}(G)$ with eigenvalues

$$
\lambda_{1}>\lambda_{2}>\cdots>\lambda_{n-1}>\lambda_{n}=0
$$

and corresponding entry-wise nonzero unit eigenvectors $v_{1}, \ldots, v_{n}$ such that

$$
A=V^{T} \Lambda V=U^{T} U,
$$

where $V^{T}=\left[v_{1}, \ldots, v_{n}\right], \Lambda=\operatorname{diag}\left(\lambda_{1}, \ldots, \lambda_{n-1}, 0\right), U=D V[1, \ldots, n-1 \mid 1, \ldots, n]$, and $D=$ $\operatorname{diag}\left(\sqrt{\lambda_{1}}, \ldots, \sqrt{\lambda_{n-1}}\right)$. Hence the rows of $U$ are orthogonal since $V$ is unitary. Let $C$ be the $(n-1) \times(2 n-1)$ matrix $C=\left[\begin{array}{ll}D & U\end{array}\right]$. Since $U$ is an entry-wise nonzero matrix, as long as each $a_{i}$ is also nonzero, the matrix

$$
C^{T} C=\left[\begin{array}{cc}
D^{2} & D U \\
U^{T} D & U^{T} U
\end{array}\right] \in \mathcal{S}\left(G \vee \bar{K}_{n-1}\right) .
$$

Further, the rows of $C$ are orthogonal and so

$$
C C^{T}=\left[\begin{array}{cccc}
\alpha_{1} & 0 & \ldots & 0 \\
0 & \alpha_{2} & \ldots & 0 \\
\vdots & & \ddots & \vdots \\
0 & 0 & \ldots & \alpha_{n-1}
\end{array}\right],
$$

where $\alpha_{i}=a_{i}^{2}+\lambda_{i}^{2}, i=1, \ldots, n-1$. Similar to the proof of the previous lemma, the spectrum of $C^{T} C$ is 0 with multiplicity $n$, and $\lambda_{0}$ with multiplicity $n-1$. This implies that $q\left(G \vee \overline{K_{n}}\right)=2$ and $M B\left(G \vee \bar{K}_{n-1}\right)=n-1$.

\section{Constructions}

Corollary 3.8 provides an infinite family of graphs in $M P([n-k, k])$ for various values of $n$ and $k$. Corollary 3.8 gives a complete characterization of graphs with $M P([n-k, k])$ for $k=2$, but not for any larger value of $k$. In this section, we consider graphs that are in $M P([n-k, k])$ but not covered in Corollary 3.8. First, we consider a direct construction of matrices corresponding to some of the graphs in Corollary 3.8.

Example 4.1. Let

$$
G=\left(K_{a_{1}} \cup K_{b_{1}} \cup K_{c_{1}}\right) \vee\left(K_{a_{2}} \cup K_{b_{2}} \cup K_{c_{2}}\right)
$$


with $a_{i}, b_{i}, c_{i}>1$, so that $q(G)=2$ and $M B(G)=k$ by Corollary 3.8. For $i=1,2$ and $t_{i} \in \mathbb{R}$ to be determined, set

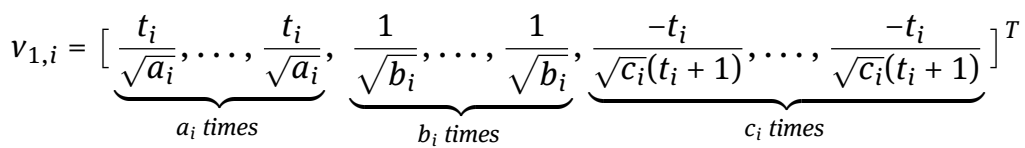

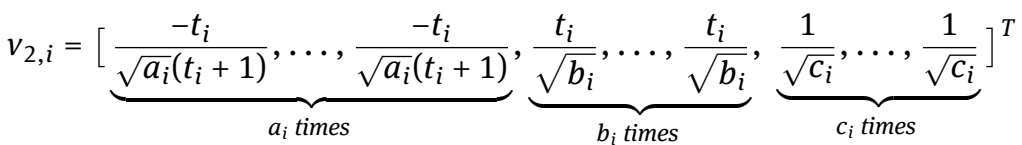

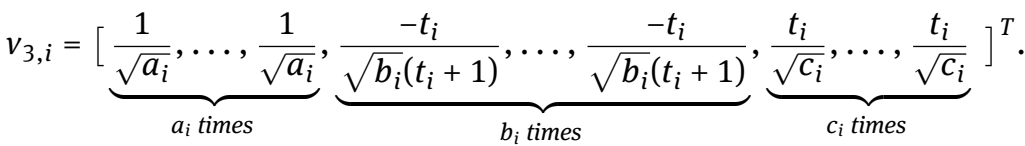

Clearly

$$
\left\|v_{1, i}\right\|=\left\|v_{2, i}\right\|=\left\|v_{3, i}\right\|=t_{i}^{2}+1+\frac{t_{i}^{2}}{\left(t_{i}+1\right)^{2}}
$$

and the three vectors are pairwise orthogonal for each $i$.

Now, form three overall vectors by concatenation, so

$$
v_{1}=\left[\begin{array}{l}
v_{1,1} \\
v_{1,2}
\end{array}\right], \quad v_{2}=\left[\begin{array}{l}
v_{2,1} \\
v_{2,2}
\end{array}\right], \quad v_{3}=\left[\begin{array}{l}
v_{3,1} \\
v_{3,2}
\end{array}\right] \text {. }
$$

These three vectors all have the same norm and are pairwise orthogonal. Let $A=I-2\left(v_{1} v_{1}^{T}+v_{2} v_{2}^{T}+v_{3} v_{3}^{T}\right)$.

Let $x, y$ be two vertices in $G$, then the $(x, y)$-entry of $A$ is given by

$$
[A]_{x, y}= \begin{cases}\frac{1}{a_{i}}\left(t_{i}^{2}+1+\frac{t_{i}^{2}}{\left(t_{i}+1\right)^{2}}\right) & \text { if } x \in V\left(K_{a_{i}}\right), \text { and } y \in V\left(K_{a_{i}}\right) ; \\ \frac{1}{b_{i}}\left(t_{i}^{2}+1+\frac{t_{i}^{2}}{\left(t_{i}+1\right)^{2}}\right) & \text { if } x \in V\left(K_{b_{i}}\right), \text { and } y \in V\left(K_{b_{i}}\right) ; \\ \frac{1}{c_{i}}\left(t_{i}^{2}+1+\frac{t_{i}^{2}}{\left(t_{i}+1\right)^{2}}\right) & \text { if } x \in V\left(K_{c_{i}}\right), \text { and } y \in V\left(K_{c_{i}}\right)\end{cases}
$$

If $x$ and $y$ are both in $K_{a_{i}} \cup K_{b_{i}} \cup K_{c_{i}}$, but not both in the same clique (induced complete graph), then $[A]_{x, y}=0$.

Assume that $x \in K_{a_{1}}$, then

$$
[A]_{x, y}= \begin{cases}\frac{1}{\sqrt{a_{1}} \sqrt{a_{2}}}\left(t_{1} t_{2}+\frac{t_{1}}{\left(t_{1}+1\right)} \frac{t_{2}}{\left(t_{2}+1\right)}+1\right) & \text { if } y \in V\left(K_{a_{2}}\right) \\ \frac{1}{\sqrt{a_{1}} \sqrt{b_{2}}}\left(t_{1}+\frac{-t_{1} t_{2}}{\left(t_{1}+1\right)}+\frac{-t_{2}}{t_{2}+1}\right) & \text { if } y \in V\left(K_{b_{2}}\right) \\ \frac{1}{\sqrt{a_{1}} \sqrt{c_{2}}}\left(\frac{-t_{1} t_{2}}{t_{2}+1}+\frac{-t_{1}}{\left(t_{1}+1\right)}+t_{2}\right) & \text { if } y \in V\left(K_{c_{2}}\right)\end{cases}
$$

If $t_{1}$ and $t_{2}$ are distinct and positive, these are all nonzero. Similarly we can show that for any $x \in K_{a_{1}} \cup K_{b_{1}} \cup K_{c_{1}}$ and any $y \in K_{a_{2}} \cup K_{b_{2}} \cup K_{c_{2}}$ that $[A]_{x, y}$ is not equal to zero. Therefore, $A \in \mathcal{S}(G)$.

Theorem 4.2. If there are $\ell$ matrices $M_{i}$ of order $k$ with the following properties

1. the rows of $M_{i}$ are orthogonal, and

2. for $i \neq j$ all the entries of $M_{i}^{T} M_{j}$ are nonzero,

then the graph

$$
G=\bigvee_{j=1}^{\ell}\left(\cup_{i=1}^{k} K_{a_{i, j}}\right)
$$

has multiplicity bipartition $[n-k, k]$ provided all $a_{i, j} \geq 1$. 
Proof. For $h=1,2, \ldots, k$, construct vectors $v_{h}$ as follows: $v_{h}=\left[v_{h, 1}, v_{h, 2}, \ldots, v_{h, \ell}\right]^{T}$, where each vector $v_{h, j}$ represents the vertices in a $\cup_{i=1}^{k} K_{a_{i, j}}$. The vertices in $K_{a_{i, j}}$ all receive the same value, namely $\frac{1}{\sqrt{a_{i, j}}}\left[M_{j}\right]_{h, i}$. Following the approach in Lemma 4.1, set $V=\left[v_{1}, \ldots, v_{k}\right]$, then $M=V^{T} V \in \mathcal{S}(G)$ and the spectrum of $M$ is $\left\{0^{(n-k)},\left\|v_{i}\right\|^{(k)}\right\}$.

In the following, we give examples of matrices that satisfy the conditions of Theorem 4.2 with order less than or equal to five, where $t>1$. Such a matrix of order 2 is

$$
M_{t}=\left[\begin{array}{cc}
1 & t \\
-t & 1
\end{array}\right] .
$$

Similarly, for orders 3, 4, and 5 we have,

$$
\begin{gathered}
M_{t}=\left[\begin{array}{ccc}
t & 1 & -\frac{t}{t+1} \\
-\frac{t}{t+1} & t & 1 \\
1 & -\frac{t}{t+1} & t
\end{array}\right], \\
M_{t}=\left[\begin{array}{cccc}
t & -1 & \frac{1}{t} & 1 \\
1 & t & -1 & \frac{1}{t} \\
\frac{1}{t} & 1 & t & -1 \\
-1 & \frac{1}{t} & 1 & t
\end{array}\right], \\
M_{s}=\left[\begin{array}{ccccc}
p & -1 & s & r & 1 \\
1 & p & -1 & s & r \\
r & 1 & p & -1 & s \\
s & r & 1 & p & -1 \\
-1 & s & r & 1 & p
\end{array}\right],
\end{gathered}
$$

with $p=\frac{-s^{2}+s-1}{s^{2}+2 s}$ and $r=\frac{s}{s+1}$.

Note that in the construction in Lemma 4.1, the numerator in the entries in the vectors come from the entries of the $3 \times 3$ matrix $M_{t}$. This method can easily be generalized.

The following lemma provides graphs with $M B(G)=3$ that are not listed in Corollary 3.8.

Lemma 4.3. Let $H=K_{\alpha, \alpha}$ be a complete bipartite graph with $2 \alpha \leq a_{1}$. Suppose

$$
G=\left(\left(K_{a_{1}} \backslash H\right) \cup K_{b_{1}}\right) \vee\left(K_{a_{2}} \cup K_{b_{2}}\right) \vee \cdots \vee\left(K_{a_{\ell}} \cup K_{b_{\ell}}\right),
$$

with $a_{i}, b_{i}>2$ and $\ell \geq 2$. Then $q(G)=2$ and $M B(G)=3$.

Proof. Let $v_{1}=\left[v_{1,1}, v_{1,2}, \ldots, v_{1, l}\right]^{T}, v_{2}=\left[v_{2,1}, v_{2,2}, \ldots, v_{2, l}\right]^{T}$, and $v_{3}=\left[v_{3,1}, v_{3,2}, \ldots, v_{3, l}\right]^{T}$, where

$$
\begin{aligned}
& v_{1, i}=[\underbrace{1, \ldots, 1}_{a_{i} \text { times }}-\underbrace{\sqrt{\frac{a_{i}}{b_{i}}} w_{i}, \ldots,-\sqrt{\frac{a_{i}}{b_{i}}} w_{i}}_{b_{i} \text { times }}]^{T} \\
& v_{2, i}=[\underbrace{w_{i}, \ldots, w_{i}}_{a_{i} \text { times }}, \underbrace{\sqrt{\frac{a_{i}}{b_{i}}}, \ldots, \sqrt{\frac{a_{i}}{b_{i}}}}_{b_{i} \text { times }}]^{T} \\
& v_{3,1}=[\underbrace{\beta, \ldots, \beta,}_{\alpha \text { times }}, \underbrace{-\beta, \ldots,-\beta}_{\alpha \text { times }}, \underbrace{0, \ldots, 0}_{a_{1}-2 \alpha \text { times }}]^{T} \\
& v_{3,2}=\left[\begin{array}{lllll}
a, & -a, & 0, & \ldots, & 0
\end{array}\right]^{T},
\end{aligned}
$$


where $\beta=\sqrt{1+w_{1}^{2}}$ and all vectors $v_{3, i}, i \geq 3$ are zero vectors. For $i>2$, if we choose the value of $w_{i}$ large enough so that $\sum_{i=2}^{\ell} a_{i}\left(1+w_{i}^{2}\right)-\alpha\left(1+w_{1}^{2}\right)>0$, then setting $a$ so that

$$
a^{2}=\frac{\sum_{i=1}^{\ell} a_{i}\left(1+w_{i}^{2}\right)-2 \alpha\left(1+w_{1}^{2}\right)}{2}
$$

results in a vector $v_{3}$ that has the same norm as vectors $v_{1}$ and $v_{2}$. The vectors $v_{1}^{T}, v_{2}^{T}$, and $v_{3}^{T}$ form orthogonal rows of a $3 \times|V(H)|$ matrix $U$, where the orthogonality of the columns of $U$ represents the edges and non-edges of $H$. Thus, $U^{T} U \in \mathcal{S}(G)$, which completes the proof.

This method can be extended to the graphs covered by Theorem 4.2.

Using a similar approach, we can also remove edges across the join operation. Define an operation $\left(K_{a_{1}} \cup\right.$ $\left.K_{b_{1}}\right) \dot{\vee}\left(K_{a_{2}} \cup K_{b_{2}}\right)$ that is the join of $K_{a_{1}} \cup K_{b_{1}}$ and $K_{a_{2}} \cup K_{b_{2}}$ with two disjoint edges removed between $K_{a_{1}}$ and $K_{a_{2}}$.

Lemma 4.4. Suppose $G=\left(\left(K_{a_{1}} \cup K_{b_{1}}\right) \dot{\vee}\left(K_{a_{2}} \cup K_{b_{2}}\right)\right) \vee \cdots \vee\left(K_{a_{\ell}} \cup K_{b_{\ell}}\right)$, with $a_{i} \geq 2$ for $i \geq 1, b_{2} \geq 2$, and $\ell \geq 2$. Then $q(G)=2$ and $M B(G)=3$.

Proof. Use the same vectors $v_{1, i}$ and $v_{2, i}$ as in the proof of Lemma 4.3. Set

$$
v_{3,1}=\left[\begin{array}{lll}
\beta, & -\beta, & 0, \ldots, 0,0, \ldots, 0
\end{array}\right]^{T},
$$

where there are $a_{1}-2$ zeros followed by $b_{1}$ zeros, and

$$
v_{3,2}=\left[\frac{\left(1+w_{1} w_{2}\right)}{\beta},-\frac{\left(1+w_{1} w_{2}\right)}{\beta}, \quad 0, \ldots, 0, \quad \frac{\left(1+w_{1} w_{2}\right)}{\beta},-\frac{\left(1+w_{1} w_{2}\right)}{\beta}, 0, \ldots, 0,\right]^{T},
$$

where there are $a_{2}-2$ zeros in the first group and $b_{2}-2$ zeros in the second group, and let $v_{3, i}=\mathbf{0}$ for $i=3, \ldots, \ell$. Let $v_{3}$ be the vector formed by concatenating $v_{3, i}$ for $i=1, \ldots, \ell$.

The norm of $v_{3}$ is $2 \beta^{2}+4 \frac{\left(1+w_{1} w_{2}\right)^{2}}{\beta^{2}}$. This is a continuous function in $\beta^{2}$ and it takes values in the interval $\left((4+2 \sqrt{2}) \sqrt{1+w_{1} w_{2}}, \infty\right)$. The norm of $v_{1}$ is at least $4+2 w_{1}^{2}+2 w_{2}^{2}$. Since it is possible to choose $w_{1}$ and $w_{2}$ so that the norm of $v_{1}$ is larger than $(4+2 \sqrt{2}) \sqrt{1+w_{1} w_{2}}$, it is also possible to choose $\beta$ so that the norm of $v_{3}$ equals $\left\|v_{1}\right\|$.

This method can also be extended to the graph in Theorem 4.2.

From Theorem 2.1 we know that $q\left(P_{n} \vee P_{n}\right)=2$ and Lemma 2.6 implies that $M B\left(P_{n} \vee P_{n}\right) \geq n-1$. We consider a related graph that achieves this same lower bound. Let $P_{n}^{2}$ be the graph on $n$ vertices labeled by $1,2, \ldots, n, 1^{\prime}, 2^{\prime}, \ldots, n^{\prime}$. Vertices $i$ and $i^{\prime}$ are adjacent for all $i \in\{1, \ldots, n\}$. If $i \in 2, \ldots, n-1$, then $i$ and $i^{\prime}$ are adjacent to vertices $i-1,(i-1)^{\prime}, i+1,(i+1)^{\prime}$. Vertices 1 and $1^{\prime}$ are adjacent to vertices 2 and $2^{\prime}$. Vertices $n$ and $n^{\prime}$ are adjacent to $n-1$ and $(n-1)^{\prime}$. Note that $P_{n}^{2}$ is the strong product of $P_{n}$ and $P_{2}$.

Lemma 4.5. For any $n, q\left(P_{n}^{2}\right)=2$ and $M B\left(P_{n}^{2}\right)=n-1$.

Proof. Order the vertices of $P_{n}^{2}$ by $\left(1,1^{\prime}, 2,2^{\prime}, \ldots, n, n^{\prime}\right)$. For $i \in\{0, \ldots, n-2\}$ let $u_{i}$ be the vector with the $(2 i+1)$ and $(2 i+2)$-entries equal to 1 , the $2 i+3$ entry equal to 2 and the $2 i+4$ entry equal to -2 , and all remaining entries set to zero. These vectors satisfy the conditions of Lemma 2.2, so $q\left(P_{n}^{2}\right)=2$ and $M B\left(P_{n}^{2}\right) \leq n-1$. The result follows since $P_{n}^{2}$ has an induced path of length $n-1$ and Lemma 2.6.

A graph is a path of cliques if its set of vertices can be partitioned into clusters, such that each cluster is a clique of size at least two, and the cliques form a path. A path of cliques whose clusters have at least two vertices can be obtained from $P_{n}^{2}$ by cloning vertices. The next result follows from Proposition 3.7.

Corollary 4.6. If $G$ is a path of cliques of size at least 4 with $k$ the longest induced path in $G$, then $q(G)=2$ and $M B(G)=k-1$. 


\section{Open Problems}

In [11] a large number of graphs are shown to admit only two distinct eigenvalues. In fact, they prove that many bipartite graphs $G$ have the property that $q(\bar{G})=2$. This gives another large and diverse family of graphs with only 2 distinct eigenvalues, and for all of these graph it is interesting to consider the multiplicity bipartition. This family includes the complements of many trees, in particular they show that $q\left(\overline{P_{n}}\right)=2$ if $n \geq 6$. The only results we have are that $M B\left(\overline{P_{6}}\right)=M B\left(\overline{P_{7}}\right)=3$.

Question 5.1. What is the multiplicity bipartition for the complement of a path on at least 8 vertices?

The graphs in $M P([n-2,2])$ have been exactly characterized (see $[7,12,13,15])$, from this characterization it can be seen that there are no trees $T$ with $M B(\bar{T})=2$.

One of the types of trees considered in [11] are denoted by $S_{m, n}^{r}$ (these are called type-one trees). The graph $S_{m, n}^{r}$ is formed by taking a path on $r$ vertices and adding $m$ leaves to one end point and $n$ leaves to the other end point. Alternately, these trees are formed by taking $K_{1, k} \cup K_{1, \ell}$ and added one additional edge to make the graph connected. If the edge is added between two leaves in $K_{1, k} \cup K_{1, \ell}$, then the resulting graph is $S_{k-1, \ell-1}^{4}$; if the edge is added between a leaf and a non-leaf then the resulting graph is either $S_{k, \ell-1}^{3}$ or $S_{k-1, \ell}^{3}$; finally, if the edge is added between two non-leaves the resulting graph is $S_{k, \ell}^{2}$.

Note that if $T=S_{m, n}^{k}$ with $k=2,3,4$, then $\bar{T}$ is formed by taking $\left(K_{1} \cup K_{m^{\prime}}\right) \vee\left(K_{1} \cup K_{n^{\prime}}\right)$ and removing a single edge across the join. In Lemma 4.4, it is shown that in some cases two edges can be removed across the join. We conjecture that it is also possible to remove a single edge across the join in many cases and achieve the multiplicity bipartition $[n-3,3]$.

Conjecture 5.1. Let $T=S_{m, n}^{k}$ with $k=2,3,4$ and $m, n>1$. Then $q(\bar{T})=2$ and $M B(\bar{T})=3$.

Corollary 3.8 gives many graphs in $M P([n-k, k])$, but it is not a characterization. Is it possible to determining the minimal (in terms of the vertex cloning) graphs in $M P([n-k, k])$, and then develop a characterization of the graphs in $M P([n-k, k])$ ?

Lemmas 4.2 and 4.3 of [16] show that graphs $K_{2,2, \ldots, 2}$ and $K_{2,2, \ldots, 2,1}$ can achieve any multiplicity bipartition $[n-k, k]$ for $k=2, \ldots,\left\lfloor\frac{n}{2}\right\rfloor$. Since all graphs $G$ with $M B(G)=2$ can be obtained from $K_{2,2, \ldots, 2}$ and $K_{2,2, \ldots, 2,1}$ by cloning vertices, and since cloning can preserve the multiplicity bipartition, this implies that if a graph achieves the multiplicity bipartition $[n-2,2]$, then it also achieves the multiplicity bipartition $[n-k, k]$ for $k>2$. Therefore, we have the following result.

Lemma 5.1. For any $n, M P([n-2,2]) \subset M P([n-k, k])$ for $k \in\{2, \ldots,\lfloor n / 2\rfloor\}$.

This raises the open question of whether or not $M P([n-k, k]) \subset M P([n-k-1, k+1])$ for larger values of $k$. Characterizing graphs with $M P([n-k, k])$ for $k>2$ (or even graphs $G$ with $M B(G)=3$ ) would answer this question partially but this is likely a harder question. We also suspect that it is possible to generalize Lemma 3.4 to show that a graph $G \vee\left(K_{S_{1}} \cup K_{S_{2}} \cup \cdots \cup K_{S_{d}}\right)$ (with the conditions stated in the Lemma) is in $M P([n-k, k])$ for all $k \geq d$. This leads to our next conjecture.

Conjecture 5.2. The complete multipartite graph $K_{k, k, \ldots, k}$ can achieve all multiplicity partitions except $[n-i, i]$ for $i<k$.

Theorem 3.9 proves for two graphs $G$ and $H$ with $q(G)=q(H)=2$ and $M B(G)=M B(H)$, that $M B(G \vee H) \leq$ $M B(G)$. We conjecture that $M B(G \vee H)=M B(G)$ and that this holds in a more general setting.

Conjecture 5.3. Let $G$ and $H$ be two graphs such that $q(G)=q(H)=2$ with $M B(G)=k_{1}$ and $M B(H)=k_{2}$, and $\mathrm{mr}_{+}(G)=k_{1}$ and $\mathrm{mr}_{+}(H)=k_{2}$. Then $q(G \vee H)=2$ with $M B(G \vee H)=k$, where $k=\max \left\{k_{1}, k_{2}\right\}$.

Lemma 2.5 states that for any graph $G$ with $q(G)=2$, we have $\operatorname{mr}_{+}(G) \leq M B(G)$, and currently we are not aware of any examples of such graphs in which this inequality is strict. However, we expect that these two graph parameters may differ in general for graphs that can achieve two distinct eigenvalues.

Question 5.2. Does there exist a graph $G$ with $M B(G)>\mathrm{mr}_{+}(G)$ ? 
Finally we would like to consider the family of strongly regular graphs. Any strongly regular graph $G$ has only three distinct eigenvalues, so $q(G) \leq 3$. Our final question is the following.

Question 5.3. Which strongly regular graphs (other than $K_{n}, K_{n, n}$, and their complements) admit only two distinct eigenvalues? Among the strongly regular graphs $G$ that admit only two distinct eigenvalues, determine $M B(G)$.

A strongly regular graph has parameters $(n, k ; a, c)$ where $n$ is the number of vertices in the graph, and each vertex has degree $k$. The number of common vertices in the neighbourhoods of two adjacent vertices is $a$, and non-adjacent vertices is $c$. If a strongly regular graph $G$ has $c=1$, then by Lemma $2.4, q(G)=3$.

Acknowledgments: The work in this paper was a joint project of the Discrete Mathematics Research Group at the University of Regina, attended by all of the authors. Dr. Adm's research was supported by the German Academic Exchange Service (DAAD) with funds from the German Federal Ministry of Education and Research (BMBF) and the People Programme (Marie Curie Actions) of the European Union's Seventh Frame-work Programme (FP7/2007-2013) under REA grant agreement No.605728 (P.R.I.M.E. - Postdoctoral Researchers International Mobility Experience) during his delegation to the University of Regina and work at University of Konstanz and revised during his work at Palestine Polytechnic University. Dr. Fallat's research was supported in part by NSERC Discovery Research Grants, Application Nos.: RGPIN-2014-06036 and RGPIN-201903934. Dr. Meagher's research was supported in part by an NSERC Discovery Research Grant, Application No.: RGPIN-03952-2018. Dr. Nasserasr's research was supported in part by an NSERC Discovery Research Grant, Application No.: RGPIN-2019-05275. Dr. Plosker's research was supported by NSERC Discovery Grant number 1174582, the Canada Foundation for Innovation (CFI) grant number 35711, and the Canada Research Chairs (CRC) Program grant number 231250. Dr. Yang's research was supported in part by an NSERC Discovery Research Grant, Application No.: RGPIN-2018-06800.

\section{References}

[1] J. Ahn, C. Alar, B. Bjorkman, S. Butler, J. Carlson, A. Goodnight, H. Knox, C. Monroek, and M.C. Wigal, Ordered multiplicity inverse eigenvalue problem for graphs on six vertices, arXiv preprint arXiv:1708.02438 2017.

[2] B. Ahmadi, F. Alinaghipour, M.S. Cavers, S. Fallat, K. Meagher, and S. Nasserasr, Minimum number of distinct eigenvalues of graphs, Electronic Journal of Linear Algebra, 26 (2013) pp. 673-691.

[3] W. Barrett, S. Butler, S.M. Fallat, H.T. Hall, L. Hogben, J.C.-H. Lin, B.L. Shader, and M. Young, The inverse eigenvalue problem of a graph: Multiplicities and minors, Journal of Combinatorial Theory, Series B (2019), https://doi.org/10.1016/j.jctb.2019.10.005.

[4] W. Barrett, S. Fallat, H.T. Hall, L. Hogben, J.C.-H. Lin, and B.L. Shader, Generalizations of the Strong Arnold Property and the Minimum Number of Distinct Eigenvalues of a Graph, Electronic Journal of Combinatorics 24 (2) (2017) pp. 2-40.

[5] W. Barrett, S. Fallat, H.T. Hall, L. Hogben, J.C.-H. Lin, and B.L. Shader, Low values of $q(G)$. To be submitted.

[6] W. Barrett, H.T. Hall, and H. van der Holst The inertia set of the join of graphs, Linear Algebra and its Applications, 434 (2011) pp. 2197-2203.

[7] W. Barrett, H. van der Holst, and R. Loewy, Graphs whose minimal rank is two, Electronic Journal of Linear Algebra, 11 (2004) pp. 258-280.

[8] B. Bjorkman, L. Hogben, S. Ponce, C. Reinhart, and T. Tranel, Applications of analysis to the determination of the minimum number of distinct eigenvalues of a graph, Pure and Applied Functional Analysis, 3 (2018) pp. 537-563.

[9] S.M. Fallat and L. Hogben, The minimum rank of symmetric matrices described by a graph: a survey, Linear Algebra and its Applications, 426 (2007) pp. 558-582.

[10] I.-J. Kim and B.L. Shader, Smith normal form and acyclic matrices, Journal of Algebraic Combinatorics, 29 (2009) pp. 63-80.

[11] R.H. Levene, P. Oblak, and H. Šmigoc, A Nordhaus-Gaddum conjecture for the minimum number of distinct eigenvalues of a graph, Linear Algebra and its Applications, 564 (2019), pp. 236-263.

[12] Z. Chen, M. Grimm, P. McMichael, and C.R. Johnson, Undirected graphs of Hermitian matrices that admit only two distinct eigenvalues, Linear Algebra and its Applications, 458 (2014) pp. 403-428.

[13] K. Meagher and I. Sciriha, Graphs that have a weighted adjacency matrix with spectrum $\left\{\lambda_{1}^{n-2}, \lambda_{1}^{2}\right\}$, arXiv preprint arXiv:1504.04178, 2015. 
[14] K.H. Monfared and B.L. Shader, The nowhere-zero eigenbasis problem for a graph, Linear Algebra and its Applications, 458 (2016) pp. 296-312.

[15] P. Oblak and H. Šmigoc, Graphs that allow all the eigenvalue multiplicities to be even, Linear Algebra and its Applications, 454 (2014) pp. 72-90.

[16] P. Oblak and H. Šmigoc, The maximum of the minimal multiplicity of eigenvalues of symmetric matrices whose pattern is constrained by a graph, Linear Algebra and its Applications, 512 (2017) pp. 48-70.

[17] T. Peters, Positive semidefinite maximum nullity and zero forcing number, Electronic Journal of Linear Algebra, 23 (2012) pp. 815-830. 